\title{
Concesiones en Salud, un Modelo Válido para la Reconstrucción y Transformación de la Red Hospitalaria en Chile
}

\author{
ROBERTO TAPIA H. ${ }^{1}$ \\ 1. Consultor para el desarrollo de Concesiones en Salud.
}

El modelo de Concesiones o Asociaciones Público Privadas ha estado siendo aplicado desde hace más de 20 años a nivel internacional. Su origen se encuentra en Inglaterra a comienzos de los 90 siendo inicialmente desarrollado por gobiernos conservadores como una forma de inyectar capitales privados para bajar el nivel de deuda pública asociado a la extensión de infraestructura pública. Luego el gobierno socialista de Tony Blair extendió su uso a diversos sectores sociales como salud, educación e incluso defensa bajo el concepto de "value for money", en una visión orientada a obtener el mejor rendimiento en el uso del dinero público. En la actualidad su uso está ampliamente difundido en España, Australia, Canadá, y en Latinoamérica en Perú, México y Chile principalmente.

El modelo implica en su forma más pura, desarrollar todas las tareas de una obra de infraestructura, diseño, construcción, operación y mantenimiento, por medio de un solo contrato con un privado. Sin embargo, existen variantes, que retienen el diseño en el estado y varían el alcance de los servicios incluidos en la operación. En todos los casos debiera optarse por la mezcla público privada más eficiente en términos de asignación de riesgo, de manera que la ejecución sea más costo efectiva. En general las Concesiones son contratos de largo plazo, 15 a 30 años, que permiten extender el pago que el estado hace al privado por la construcción y operación de una obra pública y por lo mismo disminuye la carga presupuestaria gubernamental. A su vez, la integración en un solo contrato, al menos en teoría da los incentivos adecuados al privado para que optimice todos los procesos implicados en la ejecución del contrato.

En Chile la política de Concesiones de Infraestructura Pública, aplicada al desarrollo de Infraestructura Hospitalaria, tienen como marco jurídico la Ley de Concesiones de Obras Públicas, promulgada en 1996, que sitúa en el Ministerio de Obras Públicas (MOP) de Chile el desarrollo de proyectos de este tipo. Los sectores específicos, y entre ellos el de Salud, deben solicitar la aplicación de este marco jurídico mediante la suscripción de un Convenio de Mandato que faculta al MOP para desencadenar el proceso jurídico y técnico necesario para ejecutar una Concesión.

En este marco, a partir del año 2003 se desarrolló el primer proyecto de este tipo en el ámbito sanitario chileno, el Proyecto Complejo Hospitalario Salvador Infante destinado a reponer un hospital de alta complejidad en el corazón de una de las principales áreas sanitarias (Servicios de Salud) del país. Luego de tres años de ejecución, el año 2006 y junto con

Correspondencia a:

Dr. Roberto Tapia H.

E-mail: rtapia2005@gmail.com 
la llegada de una nueva administración al Ministerio de Salud de Chile, el proyecto fue detenido en un estado de avance importante que permitía iniciar su licitación con los estudios preinversionales completos. Las autoridades de la época fundamentaron esa decisión principalmente en el excesivo riesgo que atribuían al aplicar el modelo de Concesión, nunca antes probado en Chile, a un hospital de la más alta complejidad en el sistema público. Adicionalmente, el proyecto se localizaba en el Servicio de Salud que incluye a la población de mayores ingresos de Chile, lo que llevo a plantear que existía una falta de equidad en el proyecto.

A mediados del año 2006, la mismas autoridades, decidieron dar curso a la aplicación del modelo colaboración público privada a los hospitales de Maipú y La Florida, que a esa fecha estaban desarrollados a nivel de idea, y que representaban según se señaló en su momento, una menor complejidad y mayor equidad, al ofertar servicios de salud a áreas más deprivadas del gran Santiago.

El proyecto se extendió en su ejecución hasta Noviembre de 2009 cuando fue adjudicado. En ese plazo se completaron todos los estudios preinversionales hasta la etapa de anteproyecto con diseño arquitectónico avanzado y todos los procesos propios de la aplicación del modelo de Concesiones. Debe destacarse que como parte central de este proceso en el primer semestre del 2009, se ejecutó una exitosa licitación internacional con cuatro oferentes, tres de ellos con sus casas matrices en España y otro Franco Italiano, en que se logró un ahorro sustantivo del $22 \%$ del presupuesto máximo asignado para el subsidio fijo a la construcción de los dos hospitales, con un alto nivel de detalle y calidad en los proyectos evaluados. El proyecto así concesionado se encuentra próximo a su inicio de obras físicas y constituye la primera aplicación concreta del Modelo de Concesiones a un hospital público en Chile.

En el año 2009 desde el Departamento de Concesiones del Ministerio de Salud de Chile se comenzó a desarrollar los estudios iniciales para extender la aplicación del modelo de colaboración público privada a los requerimientos de inversión del sector salud. En la práctica, se identificó que la mayor parte de los hospitales de la Región Metropolitana de Chile, que involucra a la mayor población del país ya habían completado con creces su vida útil, al mismo tiempo sus presupuestos de mantenimiento preventivo regular han sido marginales, por lo que los costos de reinversión son altos y crecientes.

Considerando estos antecedentes, en Diciembre de 2009, se firmó un nuevo Convenio de Mandato entre los Ministerios de Salud y Obras Públicas para desarrollar en el modelo de Concesiones, el que sigue vigente, la reposición de los hospitales Salvador y Geriátrico, Sótero del Río y Félix Bulnes, más la construcción de un nuevo Hospital en la zona de Quilicura y un CRS en Colina. En total estos establecimientos representan más de 2000 camas que se ofertarán a una población de 3250000 habitantes, con un volumen de inversión total cercano a los US\$ 1000 millones.

En este punto y a partir del terremoto del 27 de Febrero de 2010, se puso en evidencia la situación real de la infraestructura hospitalaria del país, la que fue afectada con la pérdida total o parcial de su capacidad operativa. De esta forma, 17 Hospitales quedaron fuera de servicio, otros 8 requerían reparaciones estructurales mayores y 54 más diversas reparaciones en sus instalaciones. En total la red asistencial perdió más de 4700 camas y un número no detallado de pabellones, servicios de apoyo y otras infraestructuras vitales para su operación. Esta situación sin duda se debe a la magnitud del terremoto que afectó al país, pero también a la antigüedad, obsolescencia de la infraestructura y bajos niveles de mantenimiento en el tiempo.

Hoy día la cartera de proyectos hospitalarios asignados por el Gobierno al modelo de Concesiones supera los 3000 millones de dólares, considerando los ya mencionados, más grandes proyectos que se encontraban próximos a su construcción como el Hospital de Antofagasta, el Gustavo Frike y Complejo Hospitalario Sur que incluye a los Hospitales Barros Luco y Exequiel González Cortés, y que fueron incluidos en el modelo por el gran volumen de financiamiento requerido para su construcción tradicional.

De esta forma, hoy día, el sector salud se 
enfrenta al mayor desafío de reposición de infraestructura que ha enfrentado en su historia, sólo comparable al impacto de los terremotos del año 39 y 60 en el sur del país. La situación se ve agravada por la creciente demanda social por atención de calidad en salud y por la cada vez más evidente situación de inequidad en el acceso y financiamiento que enfrenta la población de menores ingresos, que mayoritariamente se atiende el sector público. En el sector privado la situación no es muy diferente y está comandada por la escalada de costos y la posibilidad de que las bases de los seguros privados vean atacados su concepción del financiamiento basado en riesgos de las personas como individuos vía un posible fallo adverso del Tribunal Constitucional al uso de estas herramientas.

En el ámbito de las Concesiones en Salud, la situación no es diferente. Amplios sectores temen que el modelo de Concesiones sea sólo la primera fase de eventuales privatizaciones de la gestión de salud y no reconocen en él ninguna ventaja, aspirando a que sea el Estado el que invierta directamente en la reposición hospitalaria pendiente. En este punto creemos que los espacios para los privados están claramente delimitados por la ley chilena, reservando al estado y sus funcionarios el rol de dar atención de salud directa a los usuarios del sistema público. Por otra parte, los gremios y sindica- tos del sector salud son claramente contrarios a esta posibilidad, la que ven con razón como una amenaza a la salud pública.

Muy probablemente el futuro del modelo de APP aplicado a salud en Chile dependa de un adecuado balance entre la calidad técnica de los proyectos hospitalarios en el modelo de Concesiones y la coherencia política por parte del estado en la conducción del proceso. Para esto se requiere una visión de largo plazo, acorde con proyectos y contratos que una vez establecidos trascenderán a varios gobiernos, requiriéndose un diálogo claro con los actores relevantes, médicos, otros profesionales de la salud y funcionarios, también centrado en el logro de objetivos de largo plazo. La responsabilidad final del proceso seguirá siendo del Estado, y especialmente la responsabilidad política que ningún contrato o modelo de construcción hospitalaria puede transferir a un privado. Esta más que claro que Chile necesita urgentemente nuevos hospitales de una mayor cobertura en términos de complejidad, calidad y acceso, como resultado de su crecimiento, transición demográfica y epidemiológica y creciente espiral de expectativas por parte de los usuarios. En ese marco, el modelo de Concesiones puede ofrecer una solución práctica y factible para el sistema público, que debe ser perfeccionada con una adecuada conducción técnica y política de este complejo proceso. 